Comment raisonner pour décider : apprendre à trouver des solutions de Jean-Michel Bazin et Roger Bazin, ESF éditeur, Collection formation permanente Séminaires Mucchielli Paris, 1998, 174 p.

\title{
Hélène Dufau
}

\section{(2) OpenEdition}

\section{Journals}

Édition électronique

URL : http://journals.openedition.org/communicationorganisation/2247

DOI : 10.4000/communicationorganisation.2247

ISSN : $1775-3546$

Éditeur

Presses universitaires de Bordeaux

Édition imprimée

Date de publication : 1 mai 1999

ISSN : 1168-5549

Référence électronique

Hélène Dufau, « Comment raisonner pour décider : apprendre à trouver des solutions de Jean-Michel Bazin et Roger Bazin, ESF éditeur, Collection formation permanente - Séminaires Mucchielli Paris, 1998, 174 p. », Communication et organisation [En ligne], 15 | 1999, mis en ligne le 26 mars 2012, consulté le 01 mai 2019. URL : http://journals.openedition.org/communicationorganisation/2247 ; DOI : 10.4000/communicationorganisation.2247

Ce document a été généré automatiquement le 1 mai 2019.

(C) Presses universitaires de Bordeaux 


\title{
Comment raisonner pour décider :
} apprendre à trouver des solutions de Jean-Michel Bazin et Roger Bazin, ESF éditeur, Collection formation permanente - Séminaires Mucchielli Paris, 1998, $174 \mathrm{p}$.

\author{
Hélène Dufau
}

1 Les auteurs s'efforcent dans cet ouvrage de formation de faire converger les expériences contradictoires de la résolution individuelle et de la résolution collective de problèmes.

2 Car le monde contemporain exige de chacun, au sein de sociétés et d'organisations de plus en plus évolutifs - et par-delà l'incontournable expérience professionnelle - une importante capacité d'évolution pour inventer de nouvelles approches de la réalité, notamment dans le cadre professionnel, mettre en œuvre les énergies créatrices de tous, susciter l'enthousiasme et la participation. Cette époque de transition exige de chaque individu qu'il soit capable d'analyser les problèmes qui se posent dans son quotidien, et de chercher les pratiques les plus adaptées à son travail. La formation réelle, désormais, c'est celle qui permet d'accéder à la qualité, à des ressources de changement, d'évaluation, de critique, c'est-à-dire à une véritable évolution du travail. Pour les auteurs, le préalable à tout apprentissage pourrait donc être, dans un premier temps, d'apprendre à chercher.

3 C'est pourquoi ils proposent, dans une perspective d'autoformation à la découverte, un ensemble théorique et pratique qui devrait permettre aux formateurs d'adultes d'amener leurs apprenants à mobiliser des ressources mentales fondamentales telles que l'intuition, l'imagination, l'affectivité, le tout sous le contrôle d'un raisonnement rigoureux. Se fondant sur les apports des sciences cognitives et sur leur expérience, glanée tout aussi bien en France qu'aux États-Unis ou qu'au Japon, ils nous offrent des méthodes de 
résolution de problèmes variés : mises en relation étonnantes, représentations mentales imagées, intervention de l'intuition, et déductions convergentes. Les mécanismes mentaux utilisés pour prendre connaissance d'un énoncé, choisir des pistes de recherche, trouver des solutions, sont mis en évidence de façon pratique.

4 Les auteurs proposent, comme toujours dans cette collection, un ouvrage composé d'une partie théorique, et d'exercices gradués, accompagnés de méthodes d'animation, de groupe d'études de problèmes, ainsi que de conseils pratiques pour préparer des séances de travail. Exploitation collective ou auto-formation sont également possibles.

5 Un ouvrage à conseiller à tous ceux qui sont intéressés à l'auto-développement, en tant que formateur ou à titre individuel. 